\title{
Evaluación de la composición corporal de adultos sanos por antropometría e impedancia bioeléctrica
}

\author{
Juan Carlos Aristizábal ${ }^{1}$, María Teresa Restrepo ${ }^{2}$, Alejandro Estrada ${ }^{1}$ \\ ${ }^{1}$ Escuela de Nutrición y Dietética, Universidad de Antioquia, Medellín, Colombia \\ ${ }^{2}$ Grupo de Investigación en Alimentación y Nutrición Humana, Escuela de Nutrición y Dietética, \\ Universidad de Antioquia, Medellín, Colombia
}

Introducción. La antropometría y el análisis de la impedancia bioeléctrica o bioimpedancia son métodos de campo ampliamente utilizados en nuestro medio para la evaluación de la composición corporal; a pesar de ello, se desconoce si generan resultados comparables.

Objetivo. Comparar la composición corporal de adultos sanos evaluados por antropometría y bioimpedancia.

Materiales y métodos. Se evaluaron 70 mujeres (entre los 22 y 56 años) y 53 hombres (entre los 24 y 54 años) por el método antropométrico: ecuaciones de Durning/Womersley y Jackson/ Pollock, y por bioimpedancia: técnica pie-pie. La medición de los pliegues cutáneos se hizo con un calibrador Lange y la bioimpedancia se efectuó con una báscula Tanita TBF300. Para la comparación de los métodos se empleó la t de Student pareada, la correlación de Pearson, la diferencia de proporciones y el índice kappa.

Resultados. El porcentaje de grasa promedio en los hombres fue mayor $(p=0,000)$ por Durning/ Womersley $(25,2 \%)$ que por Jackson/Pollock $(20,1 \%)$ y bioimpedancia $(19,3 \%)$. En las mujeres, el porcentaje de grasa promedio fue mayor $(p=0,000)$ por Durning/Womersley $(36,9 \%)$ que por Jackson/Pollock $(31,0 \%)$ y bioimpedancia $(27,6 \%)$. Hubo una alta correlación entre los métodos $(r>0,77)$ pero, al aplicar el mismo punto de corte para definir la obesidad, se encontró una baja concordancia entre estos $(K<0,5)$.

Conclusión. El método antropométrico, ecuación de Durning/Womersley, estimó los porcentajes de grasa más altos y la bioimpedancia, los más bajos. Se encontraron diferencias significativas entre los métodos y entre las ecuaciones antropométricas, lo cual sugiere que sus resultados no son comparables ni intercambiables.

Palabras clave: composición corporal, índice de masa corporal, antropometría/métodos, impedancia eléctrica, adulto.

\section{Body composition assessment by anthropometry and bioelectrical impedance}

Introduction. Anthropometry and electric impedance methods are widely used for body composition assessment. However the evidence is unclear whether the results obtained from the two methods can be compared.

Objective. Two methods are compared for assessment of body composition; anthropometry and electric impedance.

Materials and methods. Body composition was measured in 70 women; aged 22 to 56 and 53 men; aged 24 to 54, using anthropometry (Durning/Womersley and Jackson/Pollock skinfolds equations) and electric impedance (foot to foot electric impedance) to obtain percentage body fat.

Results. The mean percentage body fat was significant higher with Durning/Womersley $(25.2 \%)$ than Jackson/Pollock $(20.1 \%)$ and electric impedance $(19.3 \%)$ for men $(p<0.001)$. The mean percentage body fat was significant higher with Durning/Womersley (36.9\%) than Jackson/Pollock $(31.0 \%)$ and electric impedance $(27.6 \%)$ for women $(p<0.001)$. Correlation coefficient was high when two methods were compared $(r>0.77)$ however, the concordance between methods to classify obesity subjects was low $(\mathrm{K}<0.5)$. 
Conclusions. The percentages obtained from the anthropometry method were higher than the bioelectrical impedance method. A significant difference was found between methods applied in men and women, and therefore these methods are not comparable.

Key words: body composition, body mass index, anthropometry/methods, electric impedance, adult.

Existe un interés creciente por el estudio de la composición corporal como un área del conocimiento que posibilita el análisis de las variaciones en los componentes corporales asociados a los procesos de crecimiento, envejecimiento, saludenfermedad, además de los cambios originados por el balance energético y la actividad física (1-3). Por ello, el estudio de la composición corporal es una herramienta de gran utilidad en áreas como la nutrición, la medicina, la ergonomía, la antropología física y las ciencias aplicadas al deporte.

En la actualidad, existen diversidad de modelos, métodos y técnicas para la evaluación de la composición corporal; la elección de éstos depende del objetivo del estudio, del grado de precisión y exactitud que requiera la evaluación de la composición corporal y de los recursos y medios disponibles. Así, cuando se requiere una gran precisión y exactitud se deben utilizar modelos de 3 y 4 componentes, con la aplicación de métodos de laboratorio como la densitometría, la absorciometría dual con energía de rayos $X$ (DEXA) o la hidrometría, entre otros $(4,5)$. De otro lado, en estudios de campo donde se acepta menor precisión, la evaluación de la composición corporal se realiza con base en el modelo de dos componentes, el cual fracciona el cuerpo en masa grasa y masa libre de grasa, esta última denominada masa magra por algunos autores, y mediante la aplicación de métodos rápidos y sencillos como la antropometría y la impedancia bioeléctrica o bioimpedancia $(4,5)$.

La antropometría es el método de campo de mayor uso para estimar la composición corporal

\footnotetext{
Correspondencia:

Juan Carlos Aristizábal, Departamento de Formación Académica, Escuela de Nutrición y Dietética, Universidad de Antioquia, Carrera 75 No. 65-87, bloque 44, oficina 103, Medellín, Colombia.

Teléfonos: (094) 4259219-4259232, fax: (094) 2305007. jcaristi@pijaos.udea.edu.co
}

Recibido: 03/10/06; aceptado: 28/02/07 por ser rápido, seguro y de bajo costo. Las mediciones antropométricas tienen limitantes debidas a la variabilidad que se puede presentar en un evaluador o entre evaluadores, pero cuando las mediciones son realizadas por personal bien entrenado, estas limitaciones se reducen de forma considerable (6-8).

El indicador antropométrico de mayor uso para la evaluación de los adultos es el índice de masa corporal (IMC), recomendado por la Organización Mundial de la Salud (OMS) para estudios de tamizaje de la obesidad (9). Sin embargo, varios autores han demostrado que este índice tiene poca sensibilidad para diagnosticar la obesidad por sus limitaciones para diferenciar la masa grasa y la libre de grasa del cuerpo humano $(10,11)$.

Una de las áreas de mayor interés en el estudio de la composición corporal es la asociación entre medidas corporales y la cantidad de grasa y de masa libre de grasa. Diversos investigadores han elaborado ecuaciones de predicción de la composición corporal a partir de medidas corporales para poblaciones que difieren en edad, sexo y nivel de actividad física (12-14). En nuestro medio, las ecuaciones antropométricas de Durning/Womersley y Jackson/Pollock son ampliamente utilizadas para evaluar la población adulta sana entre los 20 y 60 años de edad (15-17).

El análisis de impedancia bioeléctrica es un método para evaluar la composición corporal que se fundamenta en la conducción de la corriente eléctrica por los tejidos corporales, la cual es alta en el tejido magro donde se encuentran en mayor proporción los líquidos acuosos y electrolitos y baja, en el tejido graso. Por tanto, la impedancia bioeléctrica es inversamente proporcional al contenido de agua corporal y de masa libre de grasa $(18,19)$. El estudio de la composición corporal por medio de la bioimpedancia ha tenido gran auge porque es un método económico, rápido, no invasivo, de escasa dificultad técnica y, a diferencia del método antropométrico, no requiere 
una alta capacitación del evaluador para la aplicación de la técnica de medición (19-21).

Existen diversas técnicas para la medición de la impedancia bioeléctrica; las más comunes son mano-pie, mano-mano y pie-pie $(22,23)$. A pesar de que la técnica mano-pie tiene mayor precisión y es la recomendada por la Sociedad Europea de Nutrición Clínica $(24,25)$, la técnica pie-pie (básculas de bioimpedancia) es la más utilizada en nuestro medio por su menor costo y fácil aplicación.

Los métodos del análisis de impedancia bioeléctrica y la antropometría poseen características similares en cuanto a accesibilidad y fácil manejo, son métodos de campo doblemente indirectos que emplean ecuaciones de predicción de acuerdo con la edad, el sexo y el nivel de actividad física del individuo evaluado. Sin embargo, al comparar los porcentajes de grasa corporal estimados por ambos métodos con la densitometría como método de referencia, se han encontrado resultados contradictorios. Algunos autores han reportado una mejor correlación entre el análisis de impedancia bioeléctrica y la densitometría, otros, por el contrario, han encontrado una mejor correlación entre la antropometría y el método de referencia $(10,26)$.

El uso de la antropometría durante años ha permitido a los profesionales de la salud familiarizarse con la aplicación de diferentes ecuaciones para la predicción del porcentaje de grasa corporal. Por su parte, el análisis de impedancia bioeléctrica es un método más reciente; su aplicación con la técnica pie-pie es la de mayor uso, a pesar de que las ecuaciones de predicción incorporadas en estos equipos no son publicadas por los fabricantes por considerarlas un secreto comercial (27).

Dadas las implicaciones que tienen los resultados del estudio de la composición corporal en las acciones de promoción de la salud, tratamiento y prevención de la enfermedad y que a la fecha no se dispone en nuestro medio de estudios publicados que comparen las ecuaciones antropométricas de mayor uso con el análisis de impedancia bioeléctrica, el objetivo de este estudio es comparar los resultados de la composición corporal de adultos sanos, de ambos sexos, evaluados por el análisis de impedancia bioeléctrica técnica pie-pie y las ecuaciones antropométricas de Durning/ Womersley y Jackson/Pollock.

\section{Materiales y métodos}

La población de referencia estuvo constituida por los maestros de secundaria que desempeñaban su labor en los grados $9^{\circ}, 10^{\circ}$ y $11^{\circ}$ en establecimientos educativos oficiales y privados de la zona urbana del municipio de Medellín, en el año 2004. De esta población se obtuvo una muestra intencional de 123 personas, seleccionadas de forma aleatoria y con proporcionalidad por sexo, 70 mujeres y 53 hombres, los cuales se consideraron sanos porque en el momento del estudio no presentaron ninguna incapacidad médica para realizar su trabajo.

A los sujetos se les informó el objetivo del estudio y las pruebas por realizar, y se obtuvo el consentimiento informado de cada uno. Desde el punto de vista ético los métodos empleados son inocuos por lo que se clasifica como una investigación con riesgo mínimo, según la Resolución 8430 de 1993 del Ministerio de Salud de la República de Colombia. Así mismo, el estudio fue avalado por el Comité de Ética del Instituto Universitario de Educación Física, de la Universidad de Antioquia.

La evaluación antropométrica la realizó un nutricionista-dietista entrenado en los estándares de las técnicas de medición de uso internacional $(15,28)$. Para garantizar la confiabilidad de los datos, las mediciones se realizaron por duplicado, cuando se encontró una diferencia superior a: $0,1 \mathrm{~kg}$ en el peso corporal, $0,5 \mathrm{~cm}$ en la estatura y $1 \mathrm{~mm}$ en los pliegues cutáneos, se realizó una tercera medición (28). La estatura se midió en cm con un estadiómetro (Handac) de 1,0 mm de precisión y el peso corporal, con una báscula electrónica ( Tanita TBF300) de 0,1kg de precisión. Los pliegues cutáneos se midieron con un adipómetro (Lange) de 1,0 mm de precisión.

El porcentaje de grasa corporal por el método antropométrico se estimó a partir de la densidad corporal con la ecuación de Siri: \% grasa $=(4,95 /$ densidad) $-4,5)^{\star} 100(15,16)$. 
Para estimar la densidad corporal, se aplicaron las ecuaciones de Jackson/Pollock que emplean la sumatoria de tres pliegues cutáneos: pectoral, abdominal y muslo medio anterior para hombres: densidad $=1,1093800-0,0008267^{\star}(\Sigma 3$ pliegues $)$ $+0,0000016^{*}(\Sigma 3 \text { pliegues })^{2}-0,0002574^{*}$ (edad en años) y para mujeres tricipital, supraespinal y muslo medio anterior: densidad $=1,099421-$ $0,0009929^{*}(\Sigma 3$ pliegues $)+0,0000023^{*}(\Sigma 3$ pliegues) ${ }^{2}-0,0001392^{*}$ (edad en años) $(15,17)$.

De igual forma, se aplicó la ecuación de Durning/ Womersley para estimar la densidad corporal a partir de la sumatoria de cuatro pliegues cutáneos: bicipital, tricipital, subescapular y de cresta ilíaca densidad $=c-m * \log (\Sigma 4$ pliegues), donde $c$ y $m$ son valores que cambian por grupo de edad (16). El porcentaje de grasa corporal por el análisis de impedancia bioeléctrica se obtuvo directamente con una báscula de bioimpedancia (Tanita TBF300) siguiendo el protocolo descrito por el fabricante (27).

Para clasificar el porcentaje de grasa corporal se empleó el sistema de clasificación propuesto por Lohman que considera la obesidad cuando la grasa corporal es mayor de $25,0 \%$ en hombres y de 32,0\% en mujeres (29). La clasificación del IMC se realizó con los criterios de la OMS, así: $<18,5$ déficit de peso, $\geq 18,5 \mathrm{y}<25,0$ peso adecuado, $\geq 25 \mathrm{~kg} / \mathrm{m}^{2} \mathrm{y}<30$ sobrepeso, $y \geq 30$ obesidad (9).

El análisis de los datos se efectuó en los programas SPSS 10,0 y Epilnfo 6,04d. La descripción de los resultados se realizó con el promedio, la desviación estándar y las proporciones con sus respectivos intervalos de confianza. Para la comparación de los métodos se empleó la prueba t de Student pareada acompañada de los intervalos de confianza para la diferencia de promedios, correlación de Pearson, diferencia de proporciones e índice kappa. La significancia estadística se fijó en un valor de $p<0,05$.

\section{Resultados}

En el cuadro 1 se presentan los valores promedio con las desviaciones estándar de la edad, el peso corporal, la estatura y el IMC. Se observa que los hombres eran más altos y pesados que las
Cuadro 1. Promedios de la edad, el peso corporal, la estatura y el índice de masa corporal (IMC) en la población, según sexo.

\begin{tabular}{lcccr}
\hline Variables & \multicolumn{2}{c}{ Mujeres } & \multicolumn{2}{c}{ Hombres } \\
\cline { 2 - 5 } & Media & DE & Media & DE \\
\hline Edad & 38,3 & 8,1 & 36,1 & 7,7 \\
Peso corporal & 59,2 & 8,4 & 72,4 & 10,1 \\
Estatura & 156,0 & 5,6 & 170,1 & 5,6 \\
IMC & 24,3 & 3,5 & 25,0 & 3,0 \\
\hline
\end{tabular}

IMC: índice de masa corporal

mujeres, pero ambos grupos presentaron valores de IMC similares (cuadro 1).

En cuanto a la clasificación del peso corporal según el IMC, éste fue adecuado en $47,2 \%\left(\mathrm{IC}_{95 \%}\right.$ $33,5 ; 61,2)$ de los hombres y en $61,4 \%\left(\mathrm{IC}_{95 \%} 49,0\right.$; $72,6)$ de las mujeres. El 49,0\% $\left(\mathrm{IC}_{95 \%} 35,3 ; 63,0\right)$ de los hombres y el $27,1 \%\left(\mathrm{IC}_{95 \%} 17,5 ; 39,3\right)$ de las mujeres presentaron sobrepeso. La prevalencia de obesidad fue de $3,8 \%\left(\mathrm{IC}_{95 \%}, 0,7 ; 14,1\right)$ en hombres y de $10,0 \%\left(\mathrm{IC}_{95 \%} 4,5 ; 20,1\right)$ en las mujeres. La delgadez sólo se presentó en el 1,5\% de las mujeres.

El porcentaje de grasa corporal promedio en los hombres fue: por la ecuación de Durning/ Womersley de $25,2 \% \pm 5,3\left(\mathrm{IC}_{95 \%} 23,8 ; 26,7\right)$, por la de Jackson/Pollock de 20,1\% $\pm 6,0\left(\mathrm{IC}_{95 \%}, 18,4\right.$; $21,8)$, y por el el análisis de impedancia bioeléctrica de 19,31\% $\pm 4,8\left(\right.$ IC $\left._{95 \%} 18,0 ; 20,6\right)$. Hubo diferencias significativas entre el porcentaje de grasa promedio obtenido por la ecuación Durning/Womersley y el análisis de impedancia bioeléctrica $(p=0,000)$ y entre las ecuaciones Durning/Womersley y Jackson/Pollock $(p=0,000)$, pero no entre el análisis de impedancia bioeléctrica y la ecuación Jackson/Pollock $(p=0,127)$ (cuadro 2).

El porcentaje de grasa corporal promedio en las mujeres fue: por la ecuación de Durning/ Womersley, de $36,9 \% \pm 5,2\left(\mathrm{IC}_{95 \%} 35,7 ; 38,1\right)$, por la de Jackson/Pollock, de 31,0\% $\pm 7,1\left(\mathrm{IC}_{95 \%} 29,3\right.$; $32,7)$, y por el análisis de impedancia bioeléctrica, de $27,6 \% \pm 6,5\left(\mathrm{IC}_{95 \%} 26,1 ; 29,1\right)$. Hubo diferencias significativas $(p=0,000)$ entre el porcentaje de grasa promedio obtenido por las ecuaciones antropométricas y entre éstas y el análisis de impedancia bioeléctrica (cuadro 2). 
Cuadro 2. Comparación del porcentaje de grasa corporal promedio, estimado por el análisis de bioimpedancia (BIA) y por las ecuaciones antropométricas de Durning/Womersley (DW) y Jackson/Pollock (JP), según sexo.

\begin{tabular}{llcccc}
\hline \multirow{2}{*}{ Sexo } & Métodos & $\begin{array}{c}\text { Diferencia } \\
\text { media }\end{array}$ & \multicolumn{2}{c}{ Intervalo de confianza 95\% } & \multirow{2}{*}{ Valor $\mathbf{p}^{*}$} \\
\cline { 5 - 6 } Hombres & Inferior & Superior & &, 000 \\
& DW - BIA & 5,92 & 5,08 & 6,76 &, 000 \\
\multirow{3}{*}{ Mujeres } & DW - JP & 5,10 & 4,33 & 5,88 &, 127 \\
& JP - BIA & 0,81 & $-0,24$ & 1,88 &, 000 \\
& DW - BIA & 9,29 & 8,36 & 10,22 &, 000 \\
\hline
\end{tabular}

DW: Durning/Womersley; JP: Jackson/Pollock; BIA: bioimpedancia

* T pareada

A pesar de las diferencias encontradas entre las ecuaciones antropométricas, y entre éstas y el análisis de impedancia bioeléctrica, la correlación entre sus resultados fue alta $(r>0,77)$ y significativa $(p=0,0000$ ) en ambos sexos (cuadro 3 ).

Al aplicar el punto de corte propuesto por Lohman para definir la obesidad, a los porcentajes de grasa corporal obtenidos por las ecuaciones antropométricas y el análisis de impedancia bioeléctrica, se observó una mayor proporción de obesos por la Durning/Womersley, $79,2 \%$ de los hombres y $88,6 \%$ de las mujeres (figura 1). No se encontraron diferencias significativas entre el porcentaje de hombres clasificados como obesos por los resultados de la Jackson/Pollock y el análisis de impedancia bioeléctrica $(p=0,084)$, pero sí entre éstos y la Durning/Womersley $(p=0,001)$. Por el contrario, en las mujeres se observaron diferencias significativas en las proporciones de obesidad tanto entre las ecuaciones antropométricas como entre éstas y el análisis de impedancia bioeléctrica $(p=0,010)$.

Cuadro 3. Correlación del porcentaje de grasa corporal estimado por el análisis de bioimpedancia (BIA) y por las ecuaciones antropométricas de Durning/Womersley (DW) y Jackson/Pollock (JP), según sexo.

\begin{tabular}{lccc}
\hline Población & Método & JP & BIA \\
\hline Hombres & DW & $0,8883(0,0000)$ & $0,8200(0,0000)$ \\
$(53)$ & JP & & $0,7754(0,0000)$ \\
Mujeres & DW & $0,8450(0,0000)$ & $0,8026(0,0000)$ \\
$(70)$ & JP & & $0,8544(0,0000)$ \\
Total & DW & $0,9151(0,0000)$ & $0,8660(0,0000)$ \\
$(123)$ & JP & & $0,8881(0,0000)$ \\
\hline
\end{tabular}

Correlación de Pearson (valor de p)
De igual forma, se obtuvieron bajos valores de concordancia al utilizar los resultados de las ecuaciones antropométricas y del análisis de impedancia bioeléctrica para clasificar la obesidad. En los hombres, la concordancia entre la Durning/ Womersley y el análisis de impedancia bioeléctrica fue de 0,21, entre la Durning/Womersley y la Jackson/Pollock, de 0,41 y entre la Jackson/ Pollock y el análisis de impedancia bioeléctrica, de 0,29. En las mujeres, la concordancia entre la Durning/Womersley y el análisis de impedancia bioeléctrica fue de 0,10 , entre la Durning/ Womersley y la Jackson/Pollock, de 0,24 y entre la Jackson/Pollock y el análisis de impedancia bioeléctrica, de 0,49.

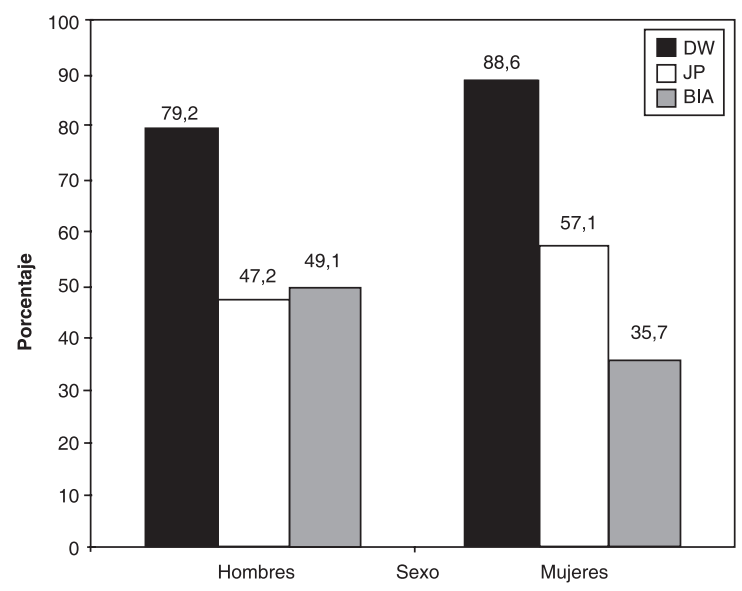

Figura 1. Comparación de la clasificación de sujetos obesos por el porcentaje de grasa corporal, estimado a partir del análisis de bioimpedancia (BIA) y las ecuaciones antropométricas de la Durning/Womersley (DW) y la de Jackson/Pollock (JP), según sexo. 


\section{Discusión}

Si bien, el objetivo del presente estudio fue evaluar el grado de comparación de la estimación de la grasa corporal de sujetos adultos por el análisis de impedancia bioeléctrica y la antropometría, llama la atención la baja proporción de sujetos clasificados como obesos según el IMC $(3,8 \%$ de los hombres y $10,0 \%$ de las mujeres) lo cual contrasta con los resultados obtenidos al evaluar la obesidad a partir del porcentaje de grasa corporal por el método antropométrico y por el análisis de impedancia bioeléctrica.

Según la ecuación de Durning/Womersley, el $88,6 \%$ de las mujeres y el $79,2 \%$ de los hombres clasificaron como obesos; con la ecuación de Jackson/Pollock, el porcentaje de obesos fue de $57,1 \%$ y $47,2 \%$ para mujeres y hombres, respectivamente; de forma similar, por el análisis de impedancia bioeléctrica, la proporción de obesos fue superior comparativamente con el IMC, 35,7\% de las mujeres y $49,1 \%$ de los hombres.

Varios autores han reportado resultados similares al comparar el IMC tanto con el método antropométrico como con el análisis de impedancia bioeléctrica y con otros métodos como la densitometría y la $\operatorname{DEXA}(10,11,30)$, lo cual ratifica la poca sensibilidad del IMC para identificar los sujetos con obesidad. La principal limitante de este indicador es la incapacidad para diferenciar los componentes magro y graso del cuerpo, ya que el problema real del obeso es el exceso de grasa y no el exceso de peso corporal $(11,31)$.

El porcentaje de grasa promedio estimado por la ecuación de Durning/Womersley fue superior al obtenido por la de Jackson/Pollock $(5,1 \%$ en hombres y $5,8 \%$ en mujeres). Lohman y colaboradores reportaron resultados similares y observaron que la ecuación de Durning/Womersley generaba porcentajes de grasa $3,5 \%$ mayores que la ecuación de Jackson/Pollock (10). Los resultados anteriores podrían atribuirse a las diferencias en los modelos de regresión de las ecuaciones, ya que las de Jackson/Pollock son cuadráticas y se ajustan a la relación curvilínea que existe entre los pliegues cutáneos y la densidad corporal $(15,17)$.
Por el contrario, las de Durning/Womersley son lineales y emplean el logaritmo de la suma de cuatro pliegues para ajustar la relación no lineal entre la densidad corporal y los pliegues cutáneos (16). De igual forma, las ecuaciones difieren en los sitios seleccionados para la medición de los pliegues cutáneos, la Durning/Womersley sólo evalúa las extremidades superiores y el tronco; la de Jackson/Pollock incluye, además, las extremidades inferiores $(15,17)$. Al respecto, como lo sugieren algunos autores, las ecuaciones de pliegues cutáneos deben considerar la distribución regional de la grasa corporal y, por tanto, deben tener en cuenta tanto pliegues del tronco como de las extremidades superiores e inferiores $(13,32)$.

Al comparar el porcentaje de grasa promedio obtenido por el método antropométrico y por el análisis de impedancia bioeléctrica en los hombres, se encontraron diferencias de 5,9\% entre el análisis de impedancia bioeléctrica y la Durning/ Womersley, y de $0,8 \%$, entre el análisis de impedancia bioeléctrica y la Jackson/Pollock; en las mujeres, de 9,3\% entre el análisis de impedancia bioeléctrica y la Durning/Womersley, y de $3,4 \%$ entre el análisis de impedancia bioeléctrica y la Jackson/Pollock.

Al respecto no se dispone de información acerca de las diferencias específicas entre el análisis de impedancia bioeléctrica por la técnica pie-pie y las ecuaciones de Durning/Womersley y Jackson/ Pollock; sólo hay reportes de que el error estándar del estimado del método antropométrico y del análisis de impedancia bioeléctrica es diferente $(10,33)$. Las diferencias entre los métodos podrían obedecer a que se fundamentan en supuestos distintos; el método antropométrico estima la densidad corporal a partir de la sumatoria de pliegues cutáneos para luego calcular los porcentajes de grasa corporal y de masa libre de grasa; por el contrario, el análisis de impedancia bioeléctrica estima el contenido de agua corporal total a partir del índice de bioimpedancia para luego calcular los porcentajes de masa libre de grasa y de grasa corporal $(15,16,34)$.

La ecuación de Durning/Womersley tiene aplicación muy difundida a pesar de que el error 
de predicción de la densidad corporal es relativamente grande (32). Al respecto, Lohman clasifica los métodos de predicción de la composición corporal según el error estándar frente al método de referencia, la densitometría (35).

A partir de esta clasificación y los errores estándar reportados en la literatura, las ecuaciones de Jackson/Pollock con un error estándar 3,5\% para hombres y de $3,9 \%$ para mujeres se clasifican como buenas; mientras que las de Durning/ Womersley con un error estándar de 4,6\% para hombres y $4,9 \%$ para mujeres se clasifican como regulares $(10,35)$.

Por su parte, el análisis de impedancia bioeléctrica por la técnica pie-pie con error estándar de $5,8 \mathrm{~kg}$ respecto a la densitometría se clasifica como deficiente $(33,35)$. De lo anterior se podría concluir que el análisis de impedancia bioeléctrica por la técnica pie-pie es de menor valor de predicción que las ecuaciones antropométricas utilizadas. Sin embargo, para recomendar la utilización de un método en particular se hace necesario realizar estudios de validación en nuestra población.

Se debe aclarar que los resultados del análisis de impedancia bioeléctrica reportados en la literatura se obtienen en su mayoría con la técnica manopie, ya que, como lo han sugerido algunos autores, con la técnica pie-pie la corriente eléctrica atraviesa principalmente el segmento inferior del cuerpo y el superior es subvalorado; además, la posición de pie puede generar una acumulación de líquidos en las extremidades inferiores y alterar los valores de bioimpedancia $(22,33)$. Éstas son algunas de las razones por las cuales la técnica mano-pie, y no la pie-pie, es la recomendada por la Sociedad Europea para el Estudio de la Nutrición Clínica $(24,25)$.

Las altas correlaciones encontradas entre el porcentaje de grasa corporal estimado por los métodos sugieren que estas metodologías presentan una tendencia similar para calcular la grasa corporal, resultados que eran de esperarse al comparar metodologías que han sido diseñadas para estimar la misma variable. Sin embargo, al aplicarles el mismo punto de corte para definir la obesidad se observaron diferencias significativas en la proporción de sujetos obesos, lo cual indica que cada método tiene una sensibilidad diferente para detectar la obesidad, hipótesis reforzada por los bajos valores de concordancia entre éstos.

Las diferencias entre los métodos y entre las ecuaciones antropométricas tienen considerables implicaciones clínicas, ya que algunos individuos se pueden clasificar como obesos, con exceso de grasa o con grasa adecuada, según el método que se utilice para estimar su composición corporal.

En conclusión, el método antropométrico con la ecuación de Durning/Womersley estimó los porcentajes de grasa más altos y el análisis de impedancia bioeléctrica por la técnica pie-pie, los más bajos. Se encontraron diferencias significativas entre los métodos y entre las ecuaciones antropométricas, lo cual sugiere que sus resultados no son comparables ni intercambiables y justificarían la aplicación de puntos de corte diferentes para definir la obesidad cuando se emplee uno u otro método. Sin embargo, debe recordarse que este estudio se realizó en una muestra de profesores de colegios públicos y privados de la ciudad y no en la población en general, lo cual demanda un mayor número de estudios en nuestra población para contrastar estos resultados.

\section{Conflicto de intereses}

Los autores del artículo dan fe de que en el desarrollo del presente trabajo no existió conflicto de intereses entre los sujetos evaluados, el ente financiador y los investigadores.

\section{Financiación}

El presente estudio forma parte de la investigación La cultura somática de los maestros en la tensión escuela adolescente, financiada por la Universidad de Antioquia y el Instituto Colombiano para el desarrollo de la Ciencia y la Tecnología, Colciencias.

\section{Referencias}

1. Seidell JC. Relationships of total and regional body fat composition to morbidity and mortality. En: Roche AF, Heymsfield SB, Lohman TG, editors. Human Body Composition. Champaign IL: Human Kinetics Publishers; 1996. p.345-53.

2. Malina RM, Bouchard C. The study of growth, maturation and performance. En: Malina RM, Bouchard C, editors. 
Growth, maturation an physical activity. Champaign IL: Human Kinetics Publishers; 1991. p.11-20.

3. Boileau RA, Horswirr CA. Body composition in sports: Measurement and applications for weight loss and gain. In: Garret WE, Kirkendall DT, editors. Exercise and sport science. Philadelphia: Lippincontt Williams and Wilkins; 2000. p.319-38.

4. Lukaski HC. Methods for assessment of human body composition: traditional and new. Am J Clin Nutr. 1987;46:537-56.

5. Wang ZM, Heshka S, Pierson RN Jr, Heymsfield SB. Systematic organization of body-composition methodology: an overview with emphasis on componentbased methods. Am J Clin Nutr. 1995;61:457-65.

6. Roche AF. Anthropometry and ultrasound. En: Roche AF, Heymsfield SB, Lohman TG, editors. Human body composition. Champaign IL: Human Kinetics Publishers; 1996. p.167-82.

7. Valtueña S, Arija V, Salas Salvadó J. Estado actual de los métodos de evaluación de la composición corporal: descripción, reproducibilidad, precisión, ámbitos de aplicación, seguridad, coste y perspectivas de futuro. Med Clin (Barc). 1996;106:624-35.

8. Urrejola P, Hodgson MI, Icaza MG. Evaluación de la composición corporal en niñas usando impedanciometría eléctrica y pliegues subcutáneos. Rev Chil Pediatr. 2001;72:26-33.

9. World Health Organization. Obesity: preventing and managing the global epidemic. In: Report of World Health Organization Consultation on Obesity. Geneva 3-5 june, 1997. Geneva: World Health Organization; 1998. p.1-276.

10. Lohman TG. Prediction equations and skinfolds, bioelectrical impedance, and body mass index. In: Lohman $T G$, editors. Advances in body composition assessment. Champaign IL: Human Kinetics Publishers; 1992. p.37-56.

11. Durnin JV, Womersley J. A comparison of the skinfold method with extent of "overweight" and various weightheight relationships in assessment of obesity. $\mathrm{Br} \mathrm{J}$ Nutr. 1977;38:271-84.

12. Lohman TG. Skinfolds and body density and their relation to body fatness: A review. Hum Biol. 1983;53: 181-225.

13. Brodie DA. Techniques of measurement of body composition. Part I. Sports Med. 1988;5:11-40.

14. Brodie DA. Techniques of measurement of body composition. Part II. Sports Med. 1988;5:74-98.

15. Jackson AS, Pollock ML. Practical assessment of body composition. Phys Sports Med. 1985;13:76-89.

16. Durnin JV, Womersley J. Body fat assessed from total body density and its estimation from skinfold thick- ness: measurements on 481 men and women aged from 16 to 72 years. Br J Nutr. 1974;32:77-97.

17. Jackson AS, Pollock ML. Generalized equations for predicting body density of men. Br J Nutr. 1978;40: 497-504.

18. Foster KR, Lukaski HC. Whole-body impedance -what does it measure? Am J Cli Nutr. 1996;64(Suppl.3): 388-96.

19. Kotler DP, Burastero S, Wang J, Pierson RN Jr. Prediction of body cell mass, fat-free mass, and total body water with bioelectrical impedance analysis: effects of race, sex and disease. Am J Clin Nutr. 1996;64(Suppl.3):489-97.

20. Casanova-Román M, Rodríguez I, Rico de Cos S, Casanova-Bellido M. Análisis de la composición corporal por parámetros antropométricos y bioeléctricos. An Pediatr (Barc). 2004;61:23-31.

21. Lupoli L, Sergi G, Coin A, Perissinotto E, Volpato E, Busetto L, et al. Body composition in underweight elderly subjects: reliability of bioelectrical impedance analysis. Clin Nutr. 2004;23:1371-80.

22. Ellis KJ. Selected body composition methods can be used in field studies. J Nutr 2001;131:1589-95.

23. Gualdi-Russo ET, Toselli S. Influence of various factors on the measurement of multifrequency bioimpedance. Homo. 2002;53:1-16.

24. Kyle UG, Bosaeus I, De Lorenzo AD, Deurenberg P, Elia M, Gómez JM, et al. Bioelectrical impedance analysis-part I: review of principles and methods. Clin Nutr. 2004;23:1226-42.

25. Kyle UG, Bosaeus I, De Lorenzo AD, Deurenberg $\mathbf{P}$, Elia M, Gómez JM, et al. Bioelectrical impedance analysis-part II: utilization in clinical practice. Clin Nutr. 2004;23:1430-53.

26. Martínez E. Validación de los métodos antropométricos para la estimación de la grasa corporal en adultos de origen latinoamericano. Lec Nutr. 2002;9:9-23.

27. TANITA. Manual de instrucciones del analizador de la composición corporal TBF300. Tokio: TANITA Corporation; 2000. p.1-40.

28. Harrison G, Buskirk ER, Carter JE, Johnston FE, Lohman TG, Pollock ML, et al. Skinfold thicknesses and measurement technique. En: Lohman TG, Roche $\mathrm{AF}$, Martorell R, editors. Antropometric standardization reference manual. Champaign IL: Human Kinetics Publishers; 1988. p.55-70.

29. Lohman TG. The prevalence of obesity in children in the United States. En: Lohman TG, editors. Advances in body composition assessment. Champaign IL: Human Kinetics Publishers; 1992. p.79-89.

30. Deurenberg $\mathbf{P}$, Andreoli $\mathbf{A}$, Borg $\mathbf{P}$, KukkonenHarjula K, De-Lorenzo A, Van-Marken L, et al. The 
validity of predicted body fat percentage from body mass index and from impedance in samples of five European populations. Eur J Clin Nutr. 2001;55:973-9.

31 Heyward VH, Stolarczyk LM. Body composition basics. En: Heyward VH, Stolarczyk LM, editors. Applied body composition assessment. Champaign IL: Human Kinetics Publishers; 1996. p.2-20.

32. Norton K. Estimación antropométrica de la grasa o adiposidad. En: Norton K, Olds T, editores. Antropométrica. Rosario: Biosystem Servicio Educativo; 2000. p.116-36.

33. Nuñez CH, Gallagher D, Visser M, Pisunyer F, Wang Z, Heymsfield SB. Bioimpedance analysis: evaluation of leg-to-leg system based on pressure contact footpad electrodes. Med Sci Sports Exerc. 1997;29:524 31.

34. National Institutes of Health Technology. NIH Consensus statement. Bioelectrical impedance analysis in body composition measurement. National Institutes of Health Technology Assessment Conference Statement. December 12-14, 1994. Nutrition. 1996;12:749-62.

35. Lohman TG. Basic concepts in body composition assessment. En: Lohman TG, editors. Advances in body composition assessment. Champaign IL: Human Kinetics Publishers; 1992. p.1-5. 